\title{
Pulling an intruder from a granular material: a novel depinning experiment
}

\author{
Yue Zhang ${ }^{1, \star}$ and Robert Behringer ${ }^{1, \star \star}$ \\ ${ }^{1}$ Department of Physics and Center for Nonlinear and Complex Systems, Duke University, Durham, North Carolina 27708, USA
}

\begin{abstract}
.
Two-dimensional impact experiments by Clark et al. [2] identified the source of inertial drag to be caused by 'collisions' with a latent force network, leading to large fluctuations of the force experienced by the impactor. These collisions provided the major drag on an impacting intruder until the intruder was nearly at rest. As a complement, we consider controlled pull-out experiments where a buried intruder is pulled out of a material, starting from rest. This provides a means to better understand the non-inertial part of the drag force, and to explore the mechanisms associated with the force fluctuations. To some extent, the pull out process is a time reversed version of the impact process. In order to visualize this pulling process, we use $2 \mathrm{D}$ photoelastic disks from which circular intruders of different radii are pulled out. We present results about the dynamics of the intruder and the structures of the force chains inside the granular system as captured by slow and high speed imaging.
\end{abstract}

\section{Introduction}

Impact and the corresponding energy loss in granular material have attracted attention for some time[1]. Recently, there has been considerable work[2][3][4][5][6]about impact in granular material. When an intruder hits a granular material, such as sand, the velocity of the intruder decreases due to the force exerted by the granular particles. Impact in granular materials is particularly common in many applications, including ballistic applications, meteorite strikes and spaceship landings.

The goal of past research has been to understand the dynamics of the impact in this complex system of granular material. There are many methods used to extract this force law empirically. A common version of the force law is

$$
m \ddot{z}=m g-f(z)-h(z) \dot{z}^{2}
$$

To observe the intruder motion and the impactor dynamics, Clark et al. used the apparatus sketched in figure 1. This consists of filling photoelastic grains between two glass plates to form a bed on which different shapes of intruders impinge. As a result, stressed regions appear brighter while stress-free areas appear darker. In the visualization of the internal stresses, the photoelastic response of the system, calibrated through intensity, provides a quantitative measure of the local pressure.

With this photoelastic method, Clark et al. established a collisional model describing the microscopic origins of the impact in granular material. In the collisional model, Clark et al. assume that the particles of granular material have formed force chains that randomly collide with the

\footnotetext{
^e-mail: yue.zhang@phy.duke.edu
}

${ }^{\star}$ e-mail: bob@phy.duke.edu

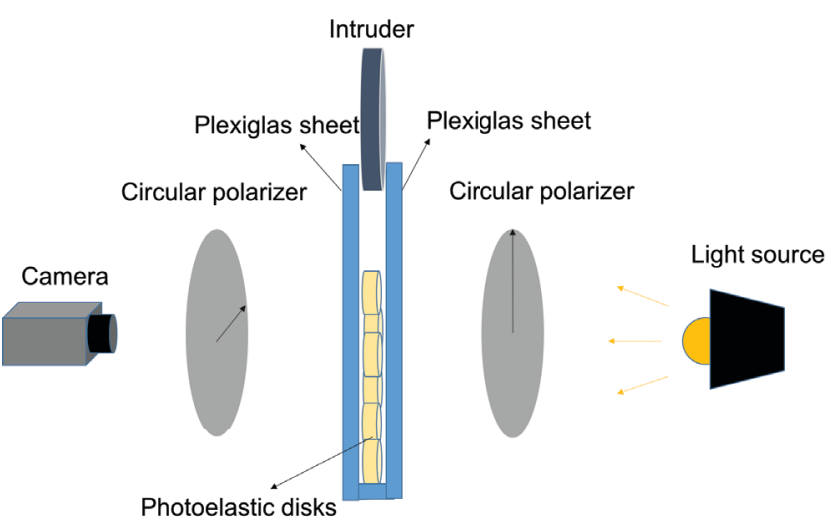

Figure 1. Schematic of apparatus of an impact experiment

intruder. They derived equations for the force exerted on the intruder, and the results match quite well with the experimental data. In this model, both the multiplicative factor in the inertial force ( $\dot{z}^{2}$ term of equation) depends on the intruder and grains properties. In addition, multiplicative fluctuations in both static and inertial forces exerted on the intruder were discovered in these experiments [2] [7] [8].

However, the characteristics of the force fluctuation still remains unknown. As a comparison and supplement, we carried out controlled pull-out experiments to explore the mechanisms associated with these fluctuations and the nature of the static force. The pull out process is, to some extent, the time reverse of the impact process. The pull out process of an object buried in a granular material also has many applications for pilings and buildings. 


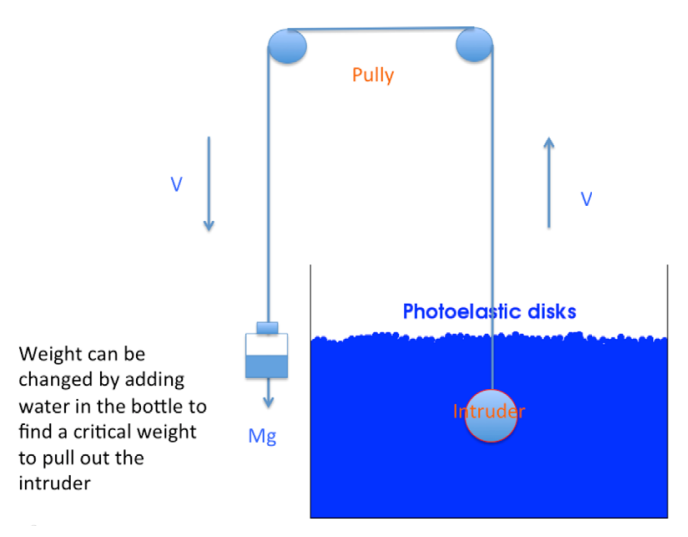

Figure 2. Images of main apparatus for pull experiment

\section{Pull Out Experiment}

For the pull out problem, we immediately come across several questions. First, what is the smallest force needed to trigger the pull out process? Second, how does the granular material respond? Third, what are the dynamics of the intruder? In order to answer these questions, we have carried out pull-out experiments using photoelastic techniques and image processing.

\subsection{Apparatus and Techniques}

The sketch above (fig.2) shows the main apparatus used in this experiment. A bidisperse mixture of about 3000 photoelastic particles (with thickness about $0.32 \mathrm{~cm}$, diameters about $0.89 \mathrm{~cm}$ and $0.56 \mathrm{~cm}$ ) are sandwiched between two sheets of transparent Plexiglas. The reason for using bidisperse particles instead of monodisperse particles is to avoid crystallization in this granular system. The distance between the two Plexiglas sheets is $0.41 \mathrm{~cm}$ so that the particles can move smoothly while loosely constrained by Plexiglas sheets. The size of each Plexiglas sheet is $122 \mathrm{~cm} \times 92 \mathrm{~cm}$. We prepare the buried intruder in each run by tipping the sheets, placing the intruder in a given position and letting particles rain back in. As a result, the particles are randomly packed. The intruder is pulled by a thread connected to a bottle whose weight can be changed by adding water to provide different pulling forces.

The recording techniques used in a pull out experiment is almost the same as in an impact experiment (fig.1), which also can visualize the force structures inside the granular system. Light from several bulbs passes through the first circular polarizer, one Plexiglas sheet, the layer of photoelastic particles, the other Plexiglas sheet and the second circular polarizer before arriving at a camera.

Then we acquire high speed video and single images. From these images we first obtain the position of the intruder. From this we track the trajectory of the intruder and derive the velocity and acceleration. The second type of information is the photoelastic visualization of force chains from the polarized image. From the force chains, we can estimate the granular structure and force acting on the intruder.
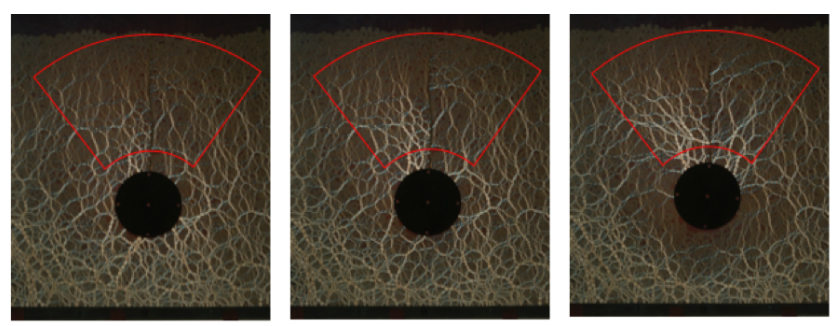

Figure 3. Force chains build up when adding load

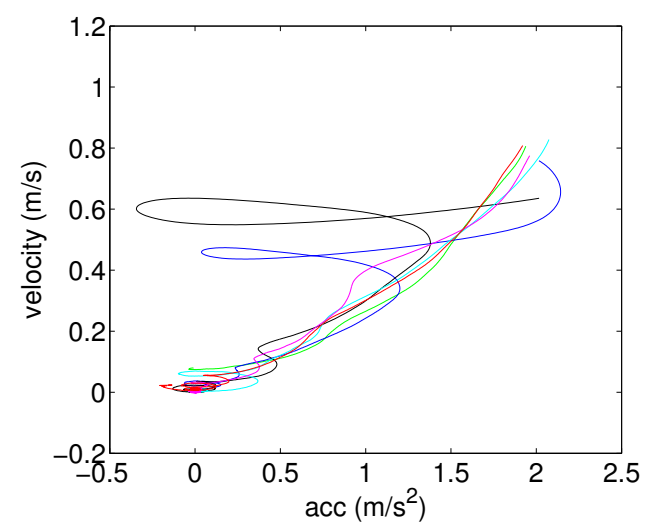

Figure 4. The graph of velocity vs. acceleration. Different colors stand for different runs with the same circular intruder $\mathrm{R}=6.4 \mathrm{~cm}$.

\subsection{Procedures and Results}

In a typical experiment, the intruder is buried at a given position in a 2D Plexiglas bed, which is filled with randomly packed photoelastic disks. Then we add load to the puller by adding small amount of water to the bottle. The process is repeated until the intruder is pulled out. As is shown in figure 3, the force chains build up step by step. We can determine the smallest pulling force needed to pull out the intruder.

Then we conduct dynamic pull experiments with the smallest pulling force. High speed video visualizes the fast process once the intruder begins to move. At first, the intruder is stuck, but then it starts to accelerate and escapes quickly from the bed. We track the intruder so that we obtain velocity and acceleration curves, as shown in figure 4. For the dynamic pull out experiment, a fast camera taking images with a speed of 1000fps is used to capture the dynamic pull process which lasts about 1 second.

As is shown in figure 5, the main part of the $v$ vs. $a$ curves can be fitted with exponential functions $v=$ $a * \exp (b t)-c$, indicating a linear relationship between velocity and acceleration as in figure 4 . The slope in figure 4 is the reciprocal of the $b$ factor in the exponential fitting function of velocity. Some loops can be found in figure 4, which is because the grains reorganize during the process creating renewed resistance to the intruder. During that time, the acceleration drops significantly.

Additionally, to visualize what is happening during the fluctuations of the acceleration, we plot space-time graphs 


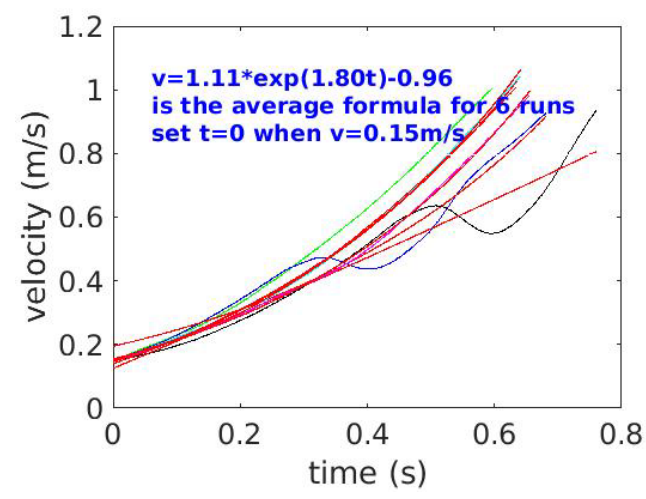

Figure 5. The graph of velocity vs. time. Different colors stand for different runs with the same circular intruder $\mathrm{R}=6.4 \mathrm{~cm}$.
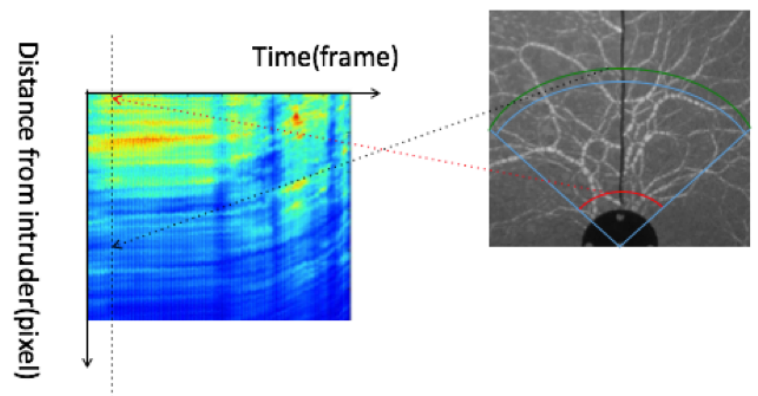

Figure 6. Space-time graph and its physical meaning.

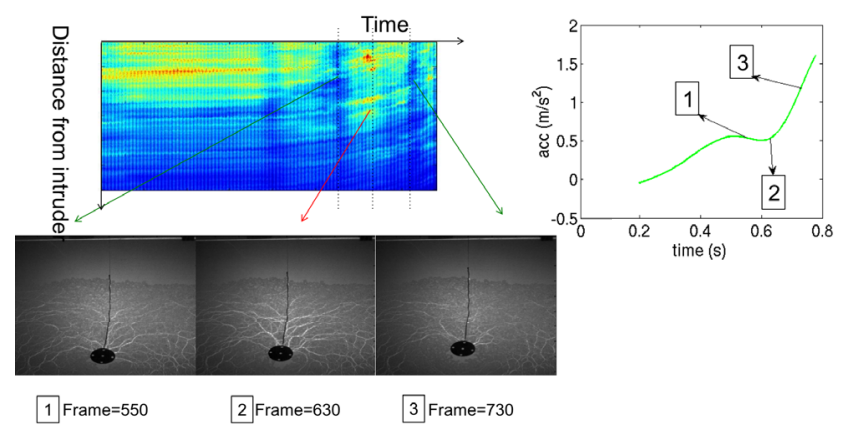

Figure 7. Discontinuities in space-time graph corresponding to fluctuations in acceleration

of the image intensity. As shown in the left graph of figure 6 , the $\mathrm{x}$-axis is time with unit of frames, and the y-axis is the distance from above the intruder in units of pixels. The right graph of figure 6 is part of a typical experimental image. We average the intensity on an arc, which gives us one point value in the space time graph.

Discontinuities in the space time graph are shown in figure 7. At those moments the force chains break and during those moments, there is a local fluctuation in acceleration.

After repeating the experiment with different circular intruders, we also find $b$, the reciprocal of the slope in the velocity vs. acceleration graph, which decreases as the ra-
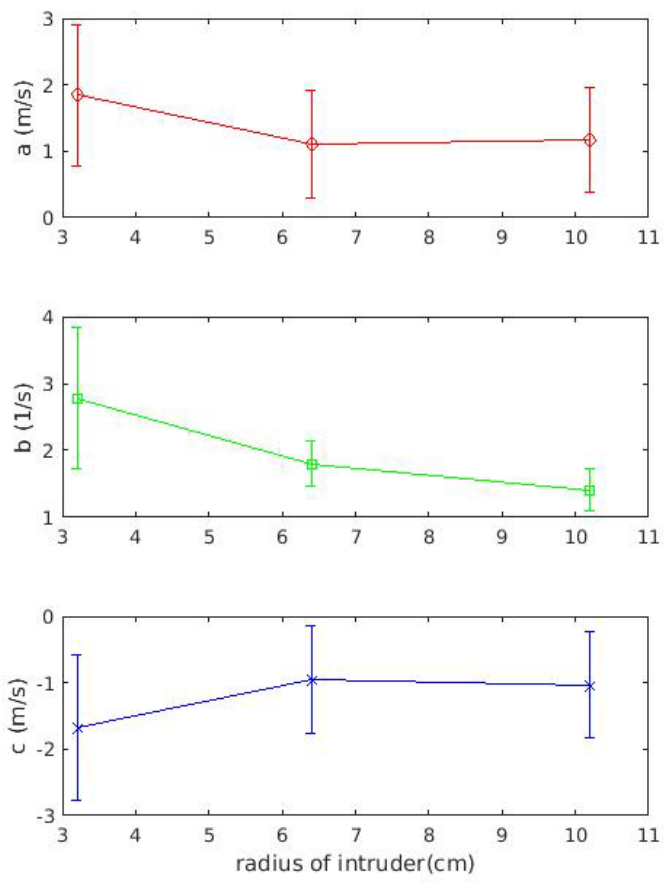

Figure 8. Fitting parameters( a, b, and c) vs. radius of intruder
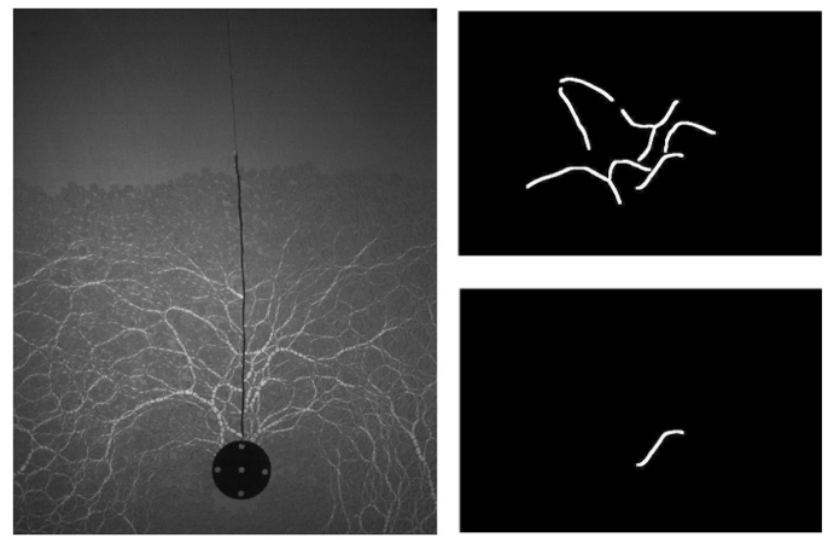

Figure 9. Extraction of single force chain: the left image is an original image from the dynamic pull-out experiments, the upper right image shows the force chains in a chosen box area above the intruder, and the lower right image shows one of those force chains in that box area.

dius increases, as shown in figure 8. However, parameters $\mathrm{a}$ and $\mathrm{c}$ in the fitting function of velocity are independent of the intruder radius. In order to understand what is happening inside the granular system, we also extract the force chains (bright parts) from high speed photoelastic images, as shown in figure 9.

Then we calculate each force chain's curvature, and obtain the distribution of curvatures. The force chain curvatures in the first and last half of the runs obey the same distribution for different intruder sizes, as shown 


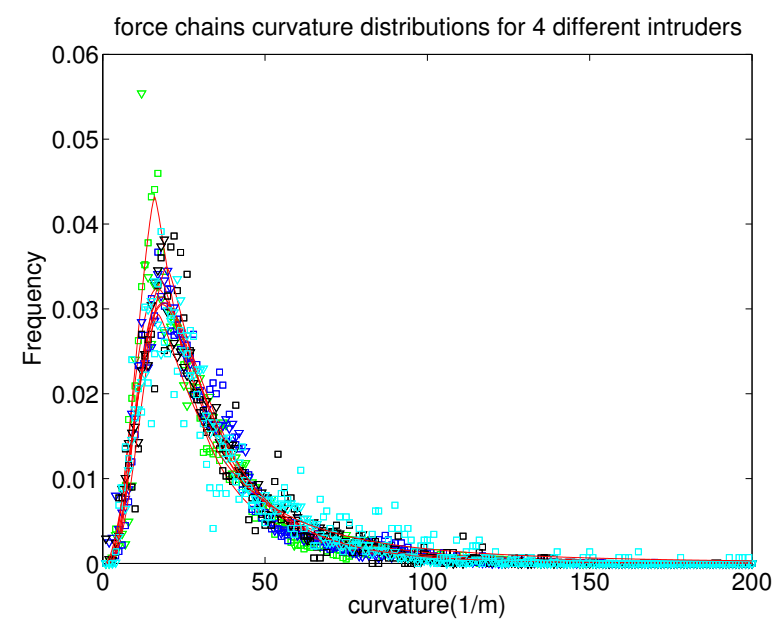

Figure 10. Force chains curvature distribution for 4 different intruders

in figure 10. Different colors stand for different intruder sizes, while triangle " $\nabla$ " and square " $\square$ " represent first and last half run, respectively. After normalization, the distributions can be collapsed into a single fitting function $y=a * \exp \left(-b *|\log (x)-c|^{d}\right)$, where $\mathrm{a}=0.03, \mathrm{~b}=1.3, \mathrm{c}=2.7$ and $d=1.9$. This curvature distribution and its effect on the stick-slips during the pull out process remains a subject for future investigation.

\section{Conclusion}

We have observed the force chain build-up in a 2D granular system during pre-pull experiments, and also investigated the dynamics of the intruder during the pull-out process. The velocity and the acceleration of the intruder follow a linear relationship, as the velocity increases exponentially with time. The $b$ factor in the exponential function decreases with the radius of the intruder. Additionally, fluctuations in the acceleration have been observed and explained through simultaneous breaking of force chains in the granular system. The curvature of each force chain can be calculated, and those curvatures are found to follow the same distribution function for different intruder sizes in both first and last half experimental run.

\section{Acknowledgements}

We would like to acknowledge NASA grant NNX15AD38G, NSF grants DMR1206351 and DMS124807, and the William M. Keck Foundation for the support of this research.

\section{References}

[1] Poncelet, J.V. Cours de M'ecanique Industrielle. Paris, 1829.

[2] Clark, A. H., et al. , "Particle scale dynamics in granular impact." Phys Rev Lett, 109, 2012.

[3] Iskander, Bless, Omidvar. Rapid Penetration into Granular Media, Visualizing the Fundamental Physics of Rapid Earth Penetration. Elsevier, 2015.

[4] Tsimring, Volfson. Powders and Grains, 2005.

[5] Katsuragi, Durian. Nature Physics, 2007.

[6] Nordstrom, K. N., et al. , "Granular dynamics during impact." Phys Rev Lett, 112, 2014.

[7] Clark, A. H., et al. , "Granular impact dynamics: Fluctuations at short time-scales." 445-448, 2013.

[8] Clark, A. H., et al. , "Collisional model for granular impact dynamics." Physical Review E 89(1), 2014. 